\title{
GAMBLER LOYALTY - A QUALITATIVE AND QUANTITATIVE INVESTIGATION
}

Catherine Prentice, Swinburne University, Australia

\begin{abstract}
Since delivering high quality service tends to improve the retention of valued customers, operators should have an understanding of how such customers react to the provision of such experiences. The current investigation explored the relationship between service quality and customer loyalty, with the latter operationalized on the basis of attitudinal and behavioural dimensions. The researchers used a combination of quantitative and qualitative methods to explore this relationship amongst gamblers whose visits to the survey casino are characterised by low, medium or high frequency. The segments that were chosen for analysis purposes were consistent with a commonly used approach to segmentation within the survey casino. The results of the quantitative study indicate that service quality explains significant variance in customer loyalty. In particular, Service environment and service delivery make substantial contributions to both attitudinal and behavioural loyalty. This is consistent with the interview findings, except that the interviews provided deeper insights into how service quality is perceived amongst customers from different groups. Such perceptions influence their intentions to return. However, when international and domestic customers are analysed separately, service quality has minimal influence on the behavioural loyalty of the latter group.
\end{abstract}

The findings of this study have the following implications: although service quality is commonly regarded as an important determinant of customer loyalty, it does not constitute the totality of a customer's judgement about an entity's overall excellence or superiority. The various service encounters experienced by customers result in differing perceptions and attitudes. An overall assessment of service quality comprises a combination of perceptions arising from each service encounter between the customer and the service provider. Since the various dimensions of service quality have differential impacts on customer loyalty, and casino managers are concerned with cost effectiveness and resource utilisation, they should focus on the aspects that most concern the loyalty and retention of particular groups. This is the first study to investigate the relationship between service quality and two-dimensional loyalty in the casino context by using the actual annual visitation to measure behavioural loyalty and by using a newly developed scale to measure casino service quality.

References available upon request 